\title{
Patient and professional experience of virtual antenatal clinics during the COVID-19 pandemic in a UK tertiary obstetric hospital: a questionnaire study
}

\author{
Lauren Quinn ${ }^{1}$, Oluwafumbi Olajide ${ }^{1}$, Marsha Green ${ }^{1}$, Hasam Sayed ${ }^{1}$, and Humera Ansar ${ }^{1}$ \\ ${ }^{1}$ University Hospitals of Leicester NHS Trust
}

October 15,2020

\begin{abstract}
Objective We evaluated patient and professional's experience with virtual antenatal clinic appointments during the COVID-19 pandemic, to determine satisfaction and enquire into the safety and quality of care received. Design, Setting, Population and Methods A total of 148 women who attended a virtual antenatal clinic appointment at our UK tertiary obstetric care centre over a two-week period provided feedback (62\% response rate). A further 37 health care professionals (HCP) delivering care in the virtual antenatal clinics participated in another questionnaire study ( $82 \%$ response rate). Main Outcome Measures and Results We showed that women were highly satisfied with the virtual clinics, with $86 \%$ rating their experience as good/very good, and this was not associated with any statistically significant differences in age, ethnicity, number of previous births or pregnancy loss $(\mathrm{es})(\mathrm{p}>0.05)$. Even though, 56\% preferred face-to-face appointments, $44 \%$ either expressed no preference or preferred virtual, and these preferences were not associated with significant differences in patient demographics ( $\mathrm{p}>0.05)$. For HCP, $67 \%$ rated their experience of virtual clinics as good/very good; $78 \%$ described their experience as the same or better than face-to-face clinics; $15 \%$ preferred virtual clinics and $44 \%$ had no preference. Importantly, $67 \%$ found it easy/very easy to adapt to virtual clinics. Over $90 \%$ of HCP agreed virtual clinics should be implemented long-term. Conclusions Our study demonstrates high satisfaction with telephone, antenatal clinics during the pandemic, which supports the transition towards widespread digitalisation of antenatal care, suited to twenty first century patients and professionals. Funding None Keywords COVID-19, Virtual clinic, antenatal, satisfaction
\end{abstract}

\section{Hosted file}

QIP_paper_final_BJOG v2.pdf available at https://authorea.com/users/367422/articles/486856patient-and-professional-experience-of-virtual-antenatal-clinics-during-the-covid-19pandemic-in-a-uk-tertiary-obstetric-hospital-a-questionnaire-study

\section{Hosted file}

Figures and tables.pdf available at https://authorea.com/users/367422/articles/486856patient-and-professional-experience-of-virtual-antenatal-clinics-during-the-covid-19pandemic-in-a-uk-tertiary-obstetric-hospital-a-questionnaire-study 